\title{
Comparative analysis of phasic left atrial strain and left ventricular posterolateral strain pattern to discriminate Fabry cardiomyopathy from other forms of left ventricular hypertrophy
}

\author{
David Frumkin ${ }^{1}$, Isabel Mattig ${ }^{1}$, Maamoun Al-Daas ${ }^{1}$, Nina-Maria Laule ${ }^{1}$, Sima \\ Canaan-kühl ${ }^{1}$, Fabian Knebel ${ }^{1}$, Karl Stangl ${ }^{2}$, and Anna Brand ${ }^{3}$ \\ ${ }^{1}$ Charité Universitätsmedizin Berlin \\ ${ }^{2}$ Charité-Universitätsmedizin Berlin \\ ${ }^{3}$ Charite Universitätsmedizin Berlin, Campus Charite Mitte
}

June 22, 2021

\begin{abstract}
Background 'Classical' echocardiographic signs of Fabry cardiomyopathy (FC), such as left ventricular hypertrophy (LVH), posterolateral strain deficiency (PLSD) and papillary muscle hypertrophy may have a limited diagnostic accuracy in clinical practice. Our aim was to evaluate the diagnostic value of left atrial (LA) strain impairment compared to 'classical' echocardiographic findings to discriminate FC. Methods In standard echocardiographic assessments, we retrospectively analyzed the diagnostic value of the "classical" red flags of FC as well as LA strain in $20 \mathrm{FC}$ patients and in 20 subjects with other causes of LVH. Receiver operating characteristic (ROC) curve analysis was performed to assess the respective diagnostic accuracy. Results FC was confirmed in 20 patients by genetic testing. In the LVH group, 12 patients were classified by biopsy to have hypertrophic cardiomyopathy, two had hypertensive heart disease, and six LVH combined with borderline myocarditis. Global and regional left ventricular (LV) strain was not significantly different between groups while LA strain was significantly impaired in FC (Left atrial reservoir strain (LASr) $19.1 \% \pm 8.4$ in FC and 25.6\% \pm 8.9 in LVH, p=0.009; left atrial conduction strain (LAScd) $-8.4 \% \pm 4.9$ in FC and $-15.9 \% \pm 8.4$ in LVH, p<0.01). LAScd, with an area under the curve (AUC) of 0.81 [95\% confidence interval (CI) 0.66-0.96] showed the highest diagnostic accuracy to discriminate FC. The PLSD pattern showed an AUC of 0.49, quantification of papillary muscle hypertrophy an AUC of 0.47. Conclusion Adding LA strain analysis to a comprehensive echocardiographic work-up of unclear LVH may be helpful to identify FC as a possible cause.
\end{abstract}

Comparative analysis of phasic left atrial strain and left ventricular posterolateral strain pattern to discriminate Fabry cardiomyopathy from other forms of left ventricular hypertrophy

David Frumkin ${ }^{1,2}$,M.D., Isabel Mattig ${ }^{1}$,M.D., Nina Laule ${ }^{1}$, Maamoun Al Daas ${ }^{1}$, Sima Canaan-Kühl ${ }^{3}$,M.D., Fabian Knebel ${ }^{1,2}$, M.D., Karl Stangl ${ }^{1,2}$, M.D., Anna Brand ${ }^{1,2}$, M.D.

1 Charite - Universitatsmedizin Berlin, corporate member of Freie Universitat Berlin and Humboldt- Universitat zu Berlin, Campus Mitte, Medizinische Klinik mit Schwerpunkt Kardiologie und Angiologie

2 DZHK (German Centre for Cardiovascular Research), partner site Berlin, Germany.

3 Charite - Universitatsmedizin Berlin, corporate member of Freie Universitat Berlin and Humboldt- Universitat zu Berlin, Campus Virchow Klinikum, Medizinische Klinik mit Schwerpunkt Nephrologie und Internistische Intensivmedizin

Short title: Left atrial strain to discriminate Fabry cardiomyopathy 


\section{Corresponding author:}

David Frumkin, M.D.

Charité - Universitätsmedizin Berlin | Campus Charité Mitte

Medizinische Klinik m. S. Kardiologie und Angiologie

Charitéplatz 1

10117 Berlin

Germany

Telephone: 004930450613194

Email:david.frumkin@charite.de

Abstract:

\section{Background}

'Classical' echocardiographic signs of Fabry cardiomyopathy (FC), such as left ventricular hypertrophy $(\mathrm{LVH})$, posterolateral strain deficiency (PLSD) and papillary muscle hypertrophy may have a limited diagnostic accuracy in clinical practice.

Our aim was to evaluate the diagnostic value of left atrial (LA) strain impairment compared to 'classical' echocardiographic findings to discriminate FC.

\section{Methods}

In standard echocardiographic assessments, we retrospectively analyzed the diagnostic value of the "classical" red flags of FC as well as LA strain in $20 \mathrm{FC}$ patients and in 20 subjects with other causes of LVH. Receiver operating characteristic (ROC) curve analysis was performed to assess the respective diagnostic accuracy.

Results

FC was confirmed in 20 patients by genetic testing. In the LVH group, 12 patients were classified by biopsy to have hypertrophic cardiomyopathy, two had hypertensive heart disease, and six LVH combined with borderline myocarditis.

Global and regional left ventricular (LV) strain was not significantly different between groups while LA strain was significantly impaired in FC (Left atrial reservoir strain (LASr) $19.1 \% \pm 8.4$ in FC and 25.6\% \pm 8.9 in $\mathrm{LVH}, \mathrm{p}=0.009$; left atrial conduction strain (LAScd) $-8.4 \% \pm 4.9$ in $\mathrm{FC}$ and $-15.9 \% \pm 8.4$ in $\mathrm{LVH}, \mathrm{p}<0.01$ ). LAScd, with an area under the curve (AUC) of 0.81 [95\% confidence interval (CI) 0.66-0.96] showed the highest diagnostic accuracy to discriminate FC. The PLSD pattern showed an AUC of 0.49 , quantification of papillary muscle hypertrophy an AUC of 0.47 .

Conclusion

Adding LA strain analysis to a comprehensive echocardiographic work-up of unclear LVH may be helpful to identify FC as a possible cause.

Keywords: Fabry Disease, cardiomyopathy, speckle tracking echocardiography, left atrial strain, left atrial deformation imaging, left ventricular strain

Introduction

Fabry Disease (FD) is a rare, X-linked lysosomal storage disease that leads to a deficient activity of the enzyme $\alpha$-galactosidase and consequently progressive accumulation of sphingolipids in multiple organs, including the heart. This results in progressive concentric left ventricular (LV) hypertrophy (LVH) ${ }^{1}$, making cardiovascular death the leading cause of mortality in patients with $\mathrm{FD}^{2,3}$. The prevalence of Fabry disease 
has been reported to be around 1\% in hypertrophic cardiomyopathy population and 1:117.000 in general population based on clinical data ${ }^{4,5}$, although underdiagnosis is common and the true prevalence may be significantly higher ${ }^{6}$. Enzyme replacement therapy (ERT) and novel chaperone-based therapy is available for FD patients ${ }^{7}$ and several treatments including modified enzymes, substrate reduction therapy and gene therapy are in development. Studies have demonstrated a benefit when ERT is initiated early in the course of disease, but efficacy is uncertain when started after the development of advanced cardiomyopathy ${ }^{8}$. Early diagnosis of cardiac involvement as well as timely and effective treatment are crucial to prevent irreversible cardiomyopathy. Guidelines currently suggest strict eligibility criteria of ERT, such as significant LVH, diastolic dysfunction, and increased indexed left atrial volume (LAVI) ${ }^{9-11}$. In late stages of FD, reduced longitudinal systolic deformation, assessed by myocardial strain, could be predominately detected in the basal and mid posterolateral left ventricular (LV) segments combined with a progressive local myocardial thinning seen by magnetic resonance imaging (MRI) ${ }^{12}$. This increase in myocyte mass, which subsequently causes LVH, is thought to be a combination of the intracellular accumulation of lipid and neurohormonal activation promoting hypertrophic activation ${ }^{1,13}$. Increase in myocyte mass is not only thought to affect ventricular walls but also the papillary muscles, causing a prominent papillary muscle often linked with FD. A prominent papillary muscle in FD becomes particularly obvious in the presence of a small left cavity due to hypertrophy ${ }^{14}$.

Impairment of phasic left atrial strain (LAS) compared to healthy controls was previously shown in FD ${ }^{15,16}$ as well as in other storage diseases featuring increased myocardial wall thickness, such as cardiac amyloidosis (CA ${ }^{17}$. These findings suggest that FD may not only cause LVH and left ventricular fibrosis but may also impact on the thin-walled left atrium (LA) with consecutive impairment of LA mechanics. However, data investigating LA function and comparing its diagnostic value to parameters of regional LV function in FD, such as the posterolateral strain deficiency (PLSD) pattern, are sparse.

In this study we aimed to describe variations in phasic LA and regional LV strain in patients with FD compared to other causes of $\mathrm{LVH}$ and to assess their respective diagnostic accuracy in discriminating patients with FD.

Methods

Study population

We retrospectively screened 51 patients with FD or LVH of other cause from our registry at the Department of cardiology at Charité - Universitätsmedizin Berlin. FD was confirmed by mutation analysis genetic testing and leukocyte $\alpha$-galactosidase activity. 20 patients had confirmed diagnosis of FD. Fabry patients were compared with a group of bioptically confirmed LVH due to other causes $(n=20)$. LVH was defined as a septal or posterior wall thickness $>11 \mathrm{~mm}$ according to the European Society of Cardiology (ESC) guidelines ${ }^{18}$. All patients with LVH in the control group underwent endomyocardial biopsy to exclude infiltrative disease. All patients obtained a standardized transthoracic echocardiographic assessment between February 2013 and July 2020. Exclusion criteria were age $<18$ years or insufficient imaging of the LA, such as foreshortening or bad acoustic window. 11 patients had to be excluded due to insufficient imaging quality. Finally, we retrospectively analyzed clinical and echocardiographic data of 20 patients diagnosed with FD and of 20 patients in the LVH group. The collection of pseudonymized medical records and the conduction of the study were approved by the institutional ethics committee (registration number EA2/194/18).

Echocardiography

A standardized transthoracic echocardiographic assessment was performed in 40 patients using a Vivid E9 (GE Vingmed, Horton, Norway) with an M5S 1.5-4.5 MHz transducer. LV dimensions, LV ejection fraction (LVEF), left ventricular global longitudinal strain (LVGLS), and LA volume index (LAVI) were analyzed in accordance with the recent recommendations of the American Society of Echocardiography (ASE) and the European Association of Cardiovascular Imaging (EACVI) ${ }^{18}$.

Analysis of phasic LA and regional LV strain 
Phasic LAS was retrospectively analyzed from a standard 2D apical four chamber

view (offline analysis, EchoPAC PC, GE Vingmed, Horton, Norway), following the recent recommendations on standardization of left atrial deformation imaging ${ }^{19}$. LAS data was obtained as previously described ${ }^{17}$ : In a QRS-triggered strain curve, with LAS set to zero at the beginning of QRS, global average LA reservoir, conduit, and contraction strain were defined as specific points: LA reservoir strain (LASr) was represented by the highest average LAS value, LA conduit strain (LAS during passive LV filling, LAScd) was calculated by average LAS value at the onset of the p-wave minus LASr. LA contraction strain (LAS during peak atrial contraction, LASct) was calculated by LAS value following maximum LA contraction minus LAS at the onset of the p-wave. In patients with atrial fibrillation $(\mathrm{n}=5)$, only LASr and LAScd were obtained, as proposed by the recent recommendations of the EACVI ${ }^{19}$. As proposed by the same recommendations, the arithmetic mean of three valid measurements was determined.

LVGLS was calculated offline as the average peak systolic longitudinal LV strain basing on LV strain analyses from three apical chamber views (17 segment LV model; EchoPAC software, GE, Vingmed, Horton, Norway). PLSD was obtained by the mean of deformation values in basal posterior and lateral segments as proposed by Kramer et al. ${ }^{12}$.

Papillary muscle area to LV area ratio (PM/LV-ratio) was obtained at a transthoracic short axis view at end diastole and the areas were manually traced as proposed by Nieman et al. ${ }^{14}$. Patients in whom only one papillary muscle was seen at this view were excluded, this applied to 16 patients.

Statistical analysis

Statistical analyses were performed using SPSS (IBM Corp. Released 2020. IBM SPSS Statistics for Macintosh Version 27.0. Armonk, NY: IBM Corp). Data were expressed as mean \pm standard deviation for continuous variables or in percentage for categorical variables. Significance of differences in clinical and echocardiographic data was calculated using the non-parametric Mann-Whitney U test for continuous variables, and the Fisher's exact test for categorical variables. Receiver operating characteristic curve (ROC) analyses were performed to compare the diagnostic value of phasic LAS and the PLSD.

Results

Clinical and echocardiographic characteristics

Of the 40 patients analyzed, 20 had FD proven by genetic testing. In the LVH group, twelve patients were classified by endomyocardial biopsy to have hypertrophic cardiomyopathy, two had hypertensive heart disease, and six expressed the pattern of LV hypertrophy combined with borderline myocarditis/myocardial inflammation. Possible confounders such as age, gender and Body mass index (BMI) showed homogenous distribution between the groups without significant differences. LV and LA geometry as well as LVEF were not different between groups. LV filling parameters such as E/A and E/e' showed slightly more advanced impairment in the LVH group. PM/LV-ratio showed no significant difference between groups (Figure1 ). All clinical and echocardiographic characteristics can be inspected in Tables 1 and 2.

Regional LV function and phasic LA strain

Global and regional LV function (LVGLS and PLSD) were not significantly different between groups (Table 2 and Figure 1). In contrast, LAS of all three phases of the atrial cycle was significantly reduced in FD compared to the LVH group (Table 2 and Figure 1). Figure 2 shows representative examples of regional LVGLS and phasic LAS analysis in a patient with FD and a patient of the LVH group.

Diagnostic value of phasic LA strain in FD

A higher diagnostic accuracy could be shown for phasic LAS impairment in discriminating FD compared to parameters such as LVGLS and PLSD in ROC analyses (Table 3 and Figure 3). LAScd and LASr, with an area under the curve (AUC) of 0.81 [95\% confidence interval (CI) 0.66-0.96] for LAScd; and 0.76 [95\% CI 0.58-0.94] for LASr respectively, showed the highest diagnostic accuracy. The PLSD, in contrast, showed an 
AUC of 0.49 . LAVI also showed a poor diagnostic value to discriminate FD with an AUC of 0.58 (95\% CI $0.35-0.81 ; \mathrm{p}=0.486)$.

Discussion

In this study, we provide a comparative assessment of phasic LAS and LV mechanics, such as LVGLS and the PLSD pattern, in patients with FD and a group with LVH due to other causes. Results showed a significantly reduced phasic LAS in patients with FD compared to the LVH groups. Beyond that, we describe a higher diagnostic value of LAS assessment, compared to that of LVGLS or PLSD, in discriminating patients with FD.

Pichette et al ${ }^{15}$ published an extensive description of LAS alterations in FD and showed a significant impairment of phasic LAS in 50 patients with FD compared to 50 healthy controls. In contrast to these findings, we compared data of FD patients to those with LVH due to other causes; infiltrative disease was excluded by endomyocardial biopsy in all patients in the LVH group, a fact that is of growing importance due to the probable underreporting of infiltrative cardiomyopathy in patients with LVH during the past years ${ }^{20,21}$. Furthermore, we compared phasic LAS to more specific LV deformation parameters in FD since studies on this topic are yet scarce in cardiomyopathies, particularly in FD.

LVGLS impairment was earlier described in FD by echocardiography and MRI. Subsequently, Kramer et al. 12 described the phenomenon of PLSD in FD cardiomyopathy, concordant with posterolateral myocardial fibrosis seen in MRI; a finding which is since then thought to be the classic echocardiographic phenotype of FD cardiomyopathy ${ }^{10}$. Our study, however, showed a high diagnostic accuracy of phasic LAS impairment in discriminating FD, but a surprisingly low diagnostic accuracy for regional LVGLS and PM/LV-ratio, a fact that could be linked to power issues of our study on the one hand, and to the inclusion of patients featuring different staged FD forms with incomplete PLSD pattern combined with unspecific PLSD pattern in the LVH group on the other hand. These findings confirm the need for more reproducible research of such 'classical' appearing phenotypes in comparison with other entities of LVH. Similarly, other LA parameters recommended for echocardiographic assessment in FD by the recent expert consensus recommendation ${ }^{10}$, such as LAVI, failed to discriminate FD as well, despite their proven correlation with phasic LAS ${ }^{22}$.

Another "classical" echocardiographic sign in FD, the PM/LV-ratio ${ }^{14}$, also failed to discriminate FD in our cohort.

In a widely underdiagnosed but treatable disease such as FD with proven benefit of treatment in early stages of disease, echocardiographic parameters yielding a higher diagnostic accuracy than the echocardiographic standard approach are urgently needed. Since Fabry disease is a systemic disease and sphingolipid accumulation is histologically proven to exceed the $\mathrm{LV}$, it may be reasonable to integrate echocardiographic parameters exceeding LV geometry and function, such as impairment of LAS, into the diagnostic algorithm (Figure 4 ).

The mechanisms of the significant impairment of LAS in FD patients shown in our study are yet not thoroughly explained. The previously described pattern of PLSD is also matter of debate; previous data by Weidemann et al and Kramer et al ${ }^{8,12}$ strongly suggest that impaired regional LV function may be caused by posterior and inferolateral LV wall thinning due to fibrosis following sphingolipid accumulation. Regarding the left atrium, impairment of LAS in many cardiomyopathies and thus also in FD could be partially explained by impaired diastolic LV function and consecutively elevated LV filling pressures, a hypothesis that was previously confirmed when assessing LA mechanics in general ${ }^{23}$ and in $\mathrm{FD}^{15,16}$. However, phasic LAS showed significant reductions in the FD group while, in contrast, other parameters of LV systolic and diastolic function, i.e. LVGLS, LAVI, E/e', were not different between groups. Furthermore, NTproBNP was significantly higher in the LVH group. These findings suggest that intrinsic LA dysfunction, rather than high filling pressures imposing on LA function, may be the leading cause of LAS impairment in this FD cohort.

There was further only a weak correlation of LV systolic and diastolic parameters with LA reservoir and conduit function, a finding that is well in line with the hypothesis of phasic LAS reductions due to intrinsic 
rather than secondary LA function. Next to our study, also data by Pichette et al suggest intrinsic LAS impairment in FD in a longitudinal speckle-tracking study ${ }^{15}$ showing that after initiation of ERT therapy LA mechanics improved, whereas LVGLS remained stable. Similarly, ${ }^{17}$ in another 'thick heart pathology', i.e. CA, concomitant LA myopathy was previously considered as well, questioning the isolated influence of systolic and diastolic LV function on impaired LA function ${ }^{17}$.

Boyd et al. previously suggested an additional atrial myopathy irrespective of $\mathrm{LVH}^{16}$, a suggestion we encourage with our results since accumulation of sphingolipids in the LA wall have been shown in autopsies ${ }^{24}$. Future prospective studies, however, assessing LA and LV dysfunction as well as tissue characterization in FD are needed to investigate whether sphingolipid accumulation into the thin-walled LA may be responsible for the observed significant mechanical LA impairment.

\section{Limitations}

Our study was performed retrospectively, with all its inherent biases. Furthermore, echocardiographic images focusing of the LA are needed for LA strain analysis. However, this is neither part of standard transthoracic image acquisition beyond LA volume and area analysis nor feasible in all patients. Therefore, it contributes to the large number of exclusions. This could be avoided in future by a prospective study design with focus on LA image acquisition.

The relatively low number of studied subjects is comparable to other Fabry disease studies ${ }^{15,16}$ due to the rare nature of disease. Because the true prevalence of infiltrative disease is reported to be strongly underestimated

20,21 , we exclusively included patients in the control group in which no findings of infiltrative disease were detected by biopsy. Our results represent a first explorative description; they need to be confirmed in larger, prospective trials. Magnetic resonance imaging was not available in this cohort but should be used in future studies for further understanding of underlying pathophysiological mechanisms.

Author contributions:

David Frumkin, M.D.: Concept/design, Data analysis/interpretation, Drafting article, Critical revision of article, Approval of article, Statistics, Data collection

Isabel Mattig, M.D.: Data collection, Critical revision of article

Nina Laule: Data collection

Mamoun Al Daas: Data collection

Sima Canaan-Kühl, M.D.: Data collection

Fabian Knebel, M.D.: Concept/design, Data analysis/interpretation, Critical revision of article, Approval of article,

Karl Stangl, M.D.: Critical revision of article, Approval of article

Anna Brand, M.D.: Concept/design, Data analysis/interpretation, Drafting article, Critical revision of article, Approval of article, Statistics, Data collection

References:

1. Linhart A, Palecek T, Bultas J, et al. New insights in cardiac structural changes in patients with Fabry's disease. Am Heart J 2000;139:1101-8.

2. Putko BN, Wen K, Thompson RB, et al. Anderson-Fabry cardiomyopathy: prevalence, pathophysiology, diagnosis and treatment. Heart Fail Rev 2015;20:179-91.

3. Mehta A, Clarke JT, Giugliani R, et al. Natural course of Fabry disease: changing pattern of causes of death in FOS - Fabry Outcome Survey. J Med Genet 2009;46:548-52. 
4. Monserrat L, Gimeno-Blanes JR, Marin F, et al. Prevalence of fabry disease in a cohort of 508 unrelated patients with hypertrophic cardiomyopathy. J Am Coll Cardiol 2007;50:2399-403.

5. Mehta A, Ricci R, Widmer U, et al. Fabry disease defined: baseline clinical manifestations of 366 patients in the Fabry Outcome Survey. Eur J Clin Invest 2004;34:236-42.

6. Spada M, Pagliardini S, Yasuda M, et al. High incidence of later-onset fabry disease revealed by newborn screening. Am J Hum Genet 2006;79:31-40.

7. Germain DP, Hughes DA, Nicholls K, et al. Treatment of Fabry's Disease with the Pharmacologic Chaperone Migalastat. N Engl J Med 2016;375:545-55.

8. Weidemann F, Niemann M, Breunig F, et al. Long-term effects of enzyme replacement therapy on fabry cardiomyopathy: evidence for a better outcome with early treatment. Circulation 2009;119:524-9.

9. Biegstraaten M, Arngrimsson R, Barbey F, et al. Recommendations for initiation and cessation of enzyme replacement therapy in patients with Fabry disease: the European Fabry Working Group consensus document. Orphanet J Rare Dis 2015;10:36.

10. Linhart A, Germain DP, Olivotto I, et al. An expert consensus document on the management of cardiovascular manifestations of Fabry disease. Eur J Heart Fail 2020;22:1076-96.

11. Ortiz A, Germain DP, Desnick RJ, et al. Fabry disease revisited: Management and treatment recommendations for adult patients. Mol Genet Metab 2018;123:416-27.

12. Kramer J, Niemann M, Liu D, et al. Two-dimensional speckle tracking as a non-invasive tool for identification of myocardial fibrosis in Fabry disease. Eur Heart J 2013;34:1587-96.

13. Shah JS, Hughes DA, Tayebjee MH, MacFadyen RJ, Mehta AB, Elliott PM. Extracellular matrix turnover and disease severity in Anderson-Fabry disease. J Inherit Metab Dis 2007;30:88-95.

14. Niemann M, Liu D, Hu K, et al. Prominent papillary muscles in Fabry disease: a diagnostic marker? Ultrasound Med Biol 2011;37:37-43.

15. Pichette M, Serri K, Page M, Di LZ, Bichet DG, Poulin F. Impaired Left Atrial Function in Fabry Disease: A Longitudinal Speckle-Tracking Echocardiography Study. J Am Soc Echocardiogr 2017;30:170-9 $\mathrm{e} 2$.

16. Boyd AC, Lo Q, Devine K, et al. Left atrial enlargement and reduced atrial compliance occurs early in Fabry cardiomyopathy. J Am Soc Echocardiogr 2013;26:1415-23.

17. Brand A, Frumkin D, Hubscher A, et al. Phasic left atrial strain analysis to discriminate cardiac amyloidosis in patients with unclear thick heart pathology. Eur Heart J Cardiovasc Imaging 2020.

18. Lang RM, Badano LP, Mor-Avi V, et al. Recommendations for cardiac chamber quantification by echocardiography in adults: an update from the American Society of Echocardiography and the European Association of Cardiovascular Imaging. Eur Heart J Cardiovasc Imaging 2015;16:233-70.

19. Badano LP, Kolias TJ, Muraru D, et al. Standardization of left atrial, right ventricular, and right atrial deformation imaging using two-dimensional speckle tracking echocardiography: a consensus document of the EACVI/ASE/Industry Task Force to standardize deformation imaging. Eur Heart J Cardiovasc Imaging 2018;19:591-600.

20. Gonzalez-Lopez E, Gallego-Delgado M, Guzzo-Merello G, et al. Wild-type transthyretin amyloidosis as a cause of heart failure with preserved ejection fraction. Eur Heart J 2015;36:2585-94.

21. Castano A, Bokhari S, Maurer MS. Unveiling wild-type transthyretin cardiac amyloidosis as a significant and potentially modifiable cause of heart failure with preserved ejection fraction. Eur Heart J 2015;36:2595-7. 
22. Blume GG, McLeod CJ, Barnes ME, et al. Left atrial function: physiology, assessment, and clinical implications. Eur J Echocardiogr 2011;12:421-30.

23. Cameli M, Lisi M, Mondillo S, et al. Left atrial longitudinal strain by speckle tracking echocardiography correlates well with left ventricular filling pressures in patients with heart failure. Cardiovasc Ultrasound 2010;8:14.

24. Sheppard MN, Cane P, Florio R, et al. A detailed pathologic examination of heart tissue from three older patients with Anderson-Fabry disease on enzyme replacement therapy. Cardiovasc Pathol 2010;19:293-301.

Figure legends:

Figure 1 : Differences of LA reservoir $(A)$, conduit $(B)$ strain, as well as of PLSD (C) and PM/LV-ratio (D) in patients with FC and LVH. (*: $p<0.05 ; \#: p=0.86 ; \xi: p=0.93 ;$ LASr: Left atrial strain rate; LAScd: Left atrial conduit strain; LA: left atrial; PLSD: posterolateral strain deficiency; PM/LV-ratio: Ratio of papillary muscle area to left ventricular area; FC: Fabry cardiomyopathy; LVH: left ventricular hypertrophy)

Figure 2 : Representative examples of LVGLS in a 17 segment model and LAS analysis in two patients of the present cohort. (A) Patient with FC featuring the PLSD pattern (yellow circle) and impairment of LASr. (B) Patient with hypertrophic cardiomyopathy also demonstrating PLSD, while LASr is less impaired. (LA: Left atrium; LV: Left ventricle; LASr: Left atrial strain rate; LAScd: Left atrial conduit strain; LASct: Left atrial contraction strain; PLSD: posterolateral strain deficiency; FC: Fabry cardiomyopathy)

Figure 3 : ROC analysis of LASr and LAScd (A) as well as of PLSD and PM/LV-ratio (B) to discriminate FC and LVH. (LASr: Left atrial strain rate; LAScd: Left atrial conduit strain; PLSD: posterolateral strain deficiency; $P M / L V$-ratio: Ratio of papillary muscle area to left ventricular area; FC: Fabry cardiomyopathy; LVH: left ventricular hypertrophy)

Figure 4 : ,Classical' echocardiographic findings when examining patients with Fabry Cardiomyopathy. (LA: Left atrium; LV: Left ventricle)

\section{Hosted file}

TABLES.docx available at https://authorea.com/users/421153/articles/527290-comparativeanalysis-of-phasic-left-atrial-strain-and-left-ventricular-posterolateral-strainpattern-to-discriminate-fabry-cardiomyopathy-from-other-forms-of-left-ventricularhypertrophy
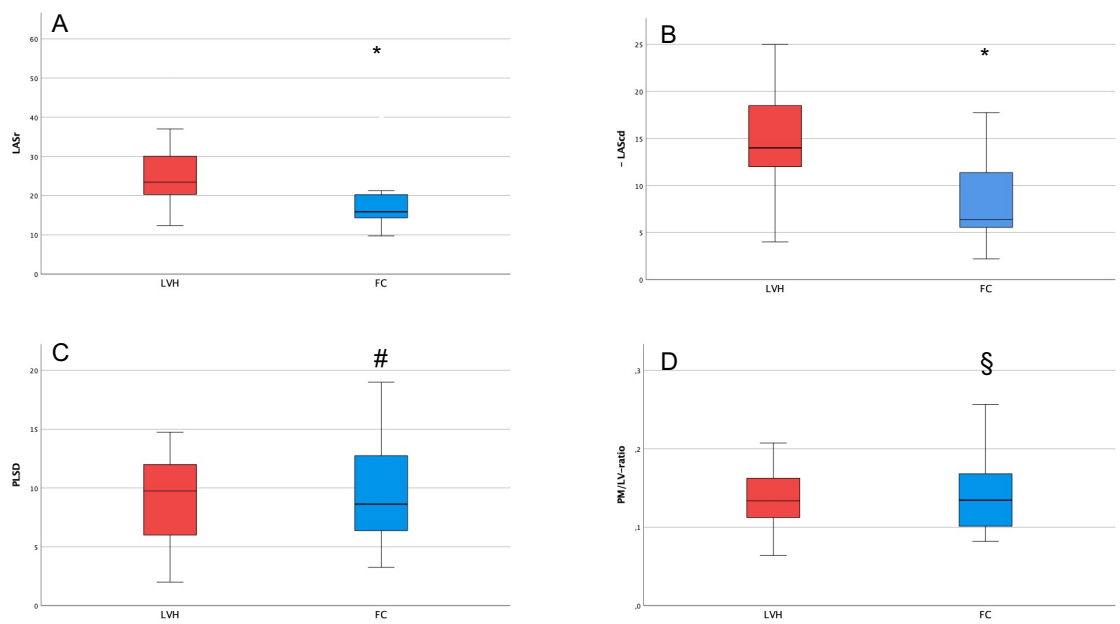

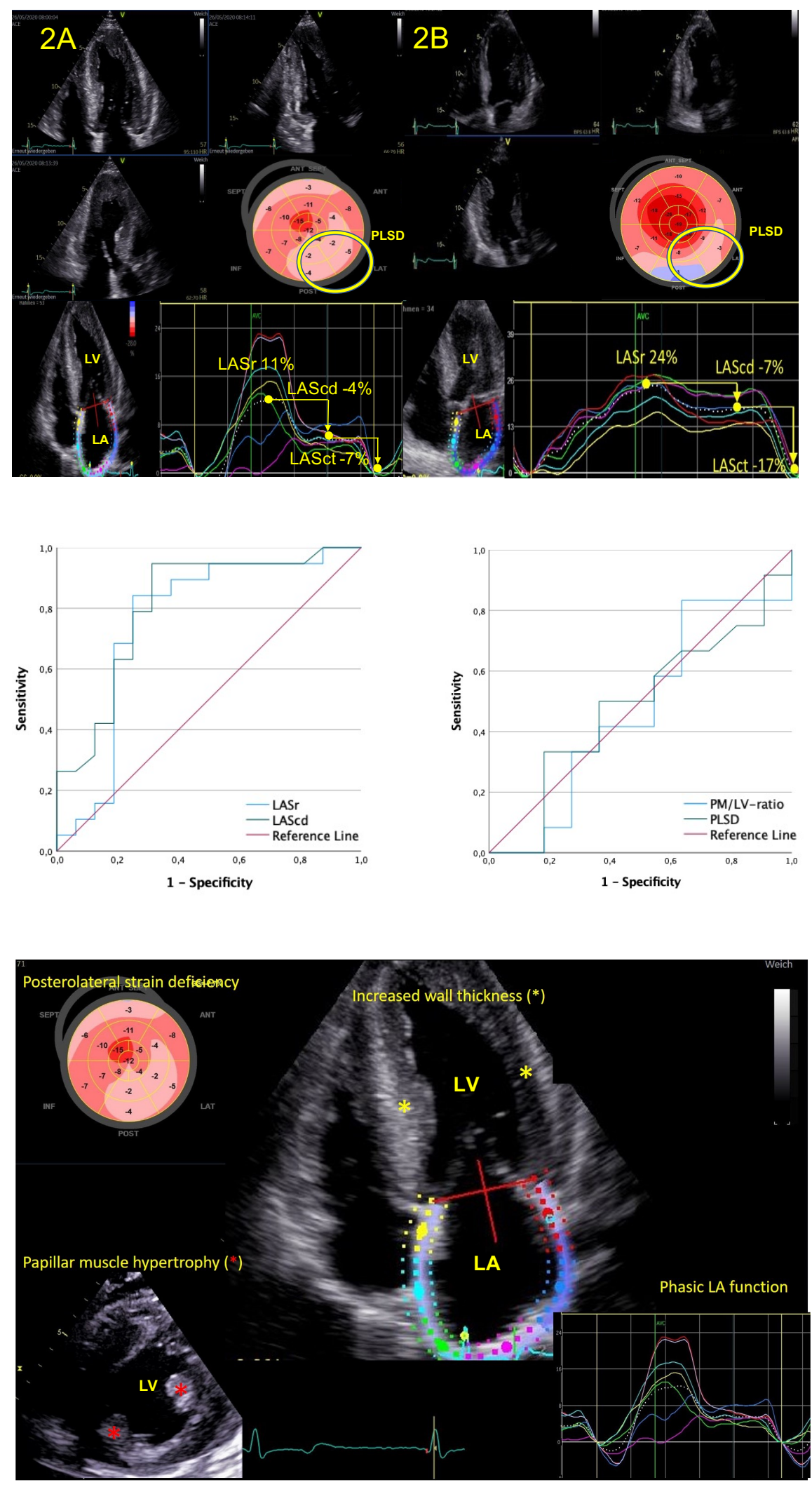